\title{
Attendance to HIV Antiretroviral Collection Clinic Appointments During COVID-19 Lockdown. A Single Center Study in Kigali, Rwanda
}

\author{
Gashema Pierre $^{1} \cdot$ Annette Uwineza ${ }^{1} \cdot$ Tafadzwa Dzinamarira $^{1,2}$ \\ Published online: 25 June 2020 \\ (c) Springer Science+Business Media, LLC, part of Springer Nature 2020
}

Globally, 78 million people have been infected with HIV and 35 million have died from AIDS-related illnesses since its inception 39 years ago [1]. In sub-Saharan Africa an estimated 25.7 million people were living with HIV (PLWH), of whom 16.4 million were taking antiretroviral therapy (ART) in the year 2018 [1]. In the same year, an estimated 470,000 people died of AIDS-related illnesses in sub-Saharan Africa [1]. In Rwanda, the Ministry of Health (MoH) led response has contributed to a stable national HIV prevalence of around 3\% in the last decade [2]. Remarkable progress has been reported in ART coverage in Rwanda with near universal coverage reported in recent surveys $[3,4]$.

The pandemic has over 7 million cases with over 400,000 deaths as of 11 June 2020 [5, 6]. An important strategy to curb transmission of the virus has been introduction of national lockdowns. In Rwanda, a national lockdown was affected on 21 March 2020 [7]; becoming the first in Africa. The lockdown banned unnecessary movements and visits outside the home and travel between different cities or districts [7]. Further, motorcycles, the most common form of transportation for the general population, were banned from ferrying passengers [7].

According to The Joint United Nations Programme on HIV and AIDS (UNAIDS) and the World Health Organization (WHO), a 6-month disruption of antiretroviral therapy (ART) during the COVID-19 pandemic in sub-Saharan Africa will increase HIV-related death rate by more than half a million [8]. This is corroborated by five well-described models of HIV epidemics which estimated that a 6-month interruption of supply of ART across the whole population

Tafadzwa Dzinamarira

anthonydzina@gmail.com

1 College of Medicine and Health Sciences, University of Rwanda, Kigali, Rwanda

2 Department of Public Health Medicine, School of Nursing and Public Health, University of KwaZulu-Natal,

Durban 4001, South Africa of people living with HIV (PLWH) on treatment would be expected to lead to an approximately twofold (from 1.87- to 2.80-fold across models) increase in HIV-related deaths over a 1 year period compared to the case with no disruption [9]. Given the recent total lockdown in Rwanda, this note presents findings from a study aimed to assess attendance to ART collection clinic appointments in the period 21 March to 30 April 2020 in Kigali, Rwanda.

We employed a cross sectional study design. The study was conducted at the University Teaching Hospital of Kigali (CHUK), ART clinic (IRB approval: EC/CHUK/036/2020). CHUK is the largest referral hospital in Rwanda. The ART clinic provides care and treatment services to approximately 2100 clients. Records from all clients with a scheduled follow-up appointment for ART collection in the period 21 March to 30 April 2020 were included. In this study, 382 records met the criteria and were available for review. Three source records were reviewed for this study namely pharmacy register, client file and linkage register. For each of these sources; clients records are linked by a unique identifier called a TracNet ID. The data abstraction form collected information on TracNet ID, client demographics (sex; age; residence), WHO clinical stage, date and result of last viral load test, current regimen, last date for ART collection and next date of appointment. Two trained health professionals abstracted the relevant data from the source records and performed quality control for accuracy during the period 21-30 May 2020. We used the chi-square test to determine association between categorical dependent and categorical independent variables.

A total of 382 participants' data was included in the analysis. 53\% were female, $23 \%$ were in the age range of $15-24$ years, while $27 \%, 20 \%$ and $29 \%$ were in the age range 25-34 years, 35-44 years and 45 years or above, respectively. Majority (88\%) resided in Kigali. Majority (91\%) achieved viral load suppression (VLS) (defined as a viral load result of less than 1000 copies $/ \mathrm{mL}$ ) based on last recorded viral load result. Less than half (48\%) had 
Table 1 Factors associated with attendance status

\begin{tabular}{|c|c|c|c|}
\hline \multirow[t]{2}{*}{ Variable } & \multicolumn{2}{|c|}{$\begin{array}{l}\text { Attendance to scheduled } \\
\text { ART collection clinic } \\
\text { appointment }\end{array}$} & \multirow[t]{2}{*}{ p-value } \\
\hline & No & Yes & \\
\hline \multirow[t]{2}{*}{ Sex, n (\%) } & $106(52)$ & $98(48)$ & 0.519 \\
\hline & $93(52)$ & $85(48)$ & \\
\hline \multicolumn{4}{|l|}{ Age in years, n (\%) } \\
\hline $15-24$ & $50(57)$ & $38(43)$ & 0.660 \\
\hline $25-34$ & $52(50)$ & $52(50)$ & \\
\hline $35-44$ & $42(54)$ & $36(46)$ & \\
\hline$\geq 45$ & $54(49)$ & $57(51)$ & \\
\hline \multicolumn{4}{|l|}{ Residence, n (\%) } \\
\hline Within Kigali & $169(50)$ & $167(50)$ & 0.040 \\
\hline Outside Kigali & $30(65)$ & $16(35)$ & \\
\hline \multicolumn{4}{|c|}{ Current drug regimen, $\mathrm{n}(\%)$} \\
\hline TDF/3TC/DTG & $43(52)$ & $40(48)$ & 0.580 \\
\hline $\mathrm{TDF} / 3 \mathrm{TC} / \mathrm{EFV}$ & $43(52)$ & $40(48)$ & \\
\hline $\mathrm{ABC} / 3 \mathrm{TC} / \mathrm{DTG}$ & $12(67)$ & $6(33)$ & \\
\hline Others & $34(50)$ & $34(50)$ & \\
\hline \multicolumn{4}{|c|}{ WHO clinical stage, n (\%) } \\
\hline 1 & $176(52)$ & $165(48)$ & 0.019 \\
\hline 2 & $7(100)$ & - & \\
\hline 3 & $8(44)$ & $10(56)$ & \\
\hline 4 & $7(78)$ & $2(22)$ & \\
\hline \multicolumn{4}{|c|}{ Viral load suppression, $\mathrm{n}(\%)$} \\
\hline No & $18(56)$ & $14(44)$ & 0.457 \\
\hline Yes & $168(54)$ & $146(46)$ & \\
\hline
\end{tabular}

attended scheduled ART collection clinic appointments during the lockdown period of 21 March to 30 April 2020.

There was an association between place of residence and attendance status $(\mathrm{p}=0.040), 50 \%$ staying within Kigali attended scheduled ART collection clinic appointments during the lockdown period compared to $35 \%$ among those living outside Kigali. There was an association between WHO clinical stage and attendance status $(\mathrm{p}=0.019), 48 \%$ in WHO clinical stage 1 attended scheduled ART collection clinic appointments during the lockdown period compared to $56 \%$ and $22 \%$ among those in WHO clinical stage 3 and 4, respectively. There was no association between attendance status with gender $(\mathrm{p}=0.159)$, age $(\mathrm{p}=0.660)$, current drug regimen $(p=0.580)$, and VLS $(p=0.457)$. Additional information about factors associated with attendance status are available in Table 1.

\section{Future Considerations}

It is well-established that without the ability to maintain consistent HIV therapy, some patients face rapid treatment failure. This calls for HIV programs to have appropriate contingency plans wherever crises that hinder PLWH from accessing care services may occur $[10,11]$. On World AIDS Day in 2016, Rwanda launched the national Differentiated Service Delivery Model (DSDM) for HIV/AIDS treatment and service provision; a strategy which includes provision for reducing the frequency of routine healthcare visits for stable, low-risk HIV patients [12]. Under the DSDM, stable patients move from monthly to quarterly visits for prescription drug pick-up and from quarterly to biannual clinic visits. This has been working well with ensuring health providers focus care and treatment efforts on higher-risk patients and new patients while stable patients spend less time and money traveling to healthcare facilities, since visits are spaced further apart. While this may be the case, there is need for advance planning to develop programs that can function during a crisis such as a nation-wide lockdown that limits people movement. The lockdown has accentuated the need for a rapid programmatic response to ensure consistent provision of care and treatment services to PLWH. Further, there is a need to strengthen effective patient tracking for individuals who miss appointments especially during a national crisis.

This study has a number of limitations. Being a singlecenter study, findings may not be generalized to map the national context. Secondly, the factors reported in this study do not show causality as there are prone to a number of confounders. However, findings provide crucial information to warrant further formative research that informs sustainable strategies to ensure continuation of care and treatment services for PLWH in similar crisis.

\section{References}

1. UNAIDS. Factsheet: global AIDS update. 2019. UNAIDS Geneva; 2019.

2. RBC. HIV Annual Report (2015-2016) 2019. https://www.rbc. gov.rw/fileadmin/user_upload/report2019/report2019/Annua 1\%2520Report\%2520for\%2520HIV\%25202015-2016.pdf.

3. ICAP. RPHIA Summary Sheet 2019. https://phia.icap.columbia. edu/wp-content/uploads/2019/10/RPHIA-Summary-Sheet_Oct2019.pdf.

4. Nsanzimana S, McArdle F, Remera E, Mulindabigwi A, Ribakare $\mathrm{M}$, Ndimubanzi $\mathrm{P}$, et al. Viral suppression in a nationwide sample of HIV-infected children on antiretroviral therapy in Rwanda. Pediatr Infect Dis J. 2019;38(2):149-51.

5. WHO. Novel coronavirus (COVID-19). World Health Organization website https://www.who.int/emergencies/diseases/novel -coronavirus-2019 Published. 2020.

6. Organization WH. Novel coronavirus (COVID-19) situation dashboard. 2020. 
7. GoR. Office of the Prime Minister: announcement on enhanced COVID-19 Prevention Measures 2020. https://primature.gov.rw/ index.php?id=2.

8. Sibeko S. UN: Sub-Saharan Africa AIDS deaths could double due to COVID-19 2020. https://www.aljazeera.com/news/2020/05/ saharan-africa-aids-deaths-double-due-covid-19-2005111630 12208.html.

9. Britta LJ, Edinah M, John S, Sherrie L. K, Andrew P. Potential effects of disruption to HIV programmes in sub-Saharan Africa caused by COVID-19: results from multiple mathematical models 2020. https://figshare.com/articles/Potential_effects_of_disru ption_to_HIV_programmes_in_sub-Saharan_Africa_caused_by_ COVID-19_results_from_multiple_mathematical_models $/ 12279$ 914.

10. Pyne-Mercier LD, John-Stewart GC, Richardson BA, Kagondu NL, Thiga J, Noshy H, et al. The consequences of post-election violence on antiretroviral HIV therapy in Kenya. AIDS Care. 2011;23(5):562-8.
11. Ssonko C, Gonzalez L, Mesic A, da Fonseca MS, Achar J, Safar $\mathrm{N}$, et al. Delivering HIV care in challenging operating environments: the MSF experience towards differentiated models of care for settings with multiple basic health care needs. J Int AIDS Soc. 2017;20(Suppl 4):21654.

12. CDC Rwanda and MSG Rwanda Interagency Team Rwanda is One of the First Countries to Launch the Differentiated HIV Care Model Nationally. https://www.cdc.gov/globalhealth/countries/ rwanda/kabeho/2017mar/dsdm.html.

Publisher's Note Springer Nature remains neutral with regard to jurisdictional claims in published maps and institutional affiliations. 